\title{
PUESTAS EN ESCENA DE OBRAS DE DRAMATURGOS ESPAÑOLES DEL SIGLO DE ORO EN LOS ÁNGELES
} (2000-2010)

\author{
PERFORMANCES OF WORKS BELONGING TO SPANISH \\ PLAYWRIGHTS OF THE SIGLO DE ORO IN LOS ANGELES \\ (2000-2010)
}

\section{John Benjamin COATES}

Gardner-Webb University/Grupo de Investigación del SELITEN@T bcoates@gardner-webb.edu

Resumen: El objetivo principal de este artículo es detallar las representaciones de obras del Siglo de Oro que se pusieron en escena en español en Los Ángeles durante los años 2000-2010.

Abstract: The main purpose of this article is to describe the performances of works belonging to the Spanish Siglo de Oro that occurred during the years 2000-2010 in Los Angeles.

Palabras clave: Teatro. Siglo de Oro. Español. Los Ángeles. 2000-2010.

Key words: Theater. Siglo de Oro. Spanish. Los Angeles. 2000-2010. 


\section{INTRODUCCIÓN}

En mi tesis de doctorado, El teatro representado en español en Los Ángeles durante los años 2000-2010, dirigida por el Dr. José Romera Castillo y que se inserta dentro de las actividades del Centro de Investigación de Semiótica Literaria, Teatral y Nuevas Tecnologías, SELITEN@T ${ }^{1}$, se constata que durante los años estudiados, hubo 259 funciones de teatro representadas en español en la ciudad con 1.785 representaciones de 182 títulos distintos (Coates, 2012: 707). También se expone que 145 autores escribieron las 182 obras que se pusieron en escena. Una observación que sobresale en este estudio es que, aunque se desconoce el origen de quince de los autores representados, los 130 autores restantes son de 25 países distintos (España y todos los países hispanohablantes de América menos Bolivia, Honduras, Nicaragua, Panamá y Paraguay). México es el país que tiene el número más alto de autores representados con 35 (el 24,14\%), seguido por España con 22 autores (el 15,17\%), según Coates (2012: 826). El propósito de este estudio es detallar las puestas en escena de las obras de algunos de estos autores españoles, los que escribieron durante el Siglo de Oro y explicar su importancia dentro del teatro representado en español en Los Ángeles en la primera década del siglo XXI.

Este trabajo, que se inserta dentro de las actividades del SELITEN@T (http://www.uned.es/centro-investeigacion-SELITEN@T/index2.html), es una continuación de la sección monográfica que aparece en la revista Signa, número 15 (http://cervantesvirtual.com/servlet/SirveObras/ 05818507811614984197857/index.htm), en la sección monográfica «Puestas en escena de nuestro teatro áureo en algunas ciudades españolas durante los siglos XIX y XX» (2006: 11-186). El profesor José Romera Castillo detalla una gran parte de estas en su libro, Pautas para la investigación del teatro español y sus puestas en escena (2011: 141-184). En las conclusiones del presente artículo, se hacen las conexiones apropiadas entre estos estudios.

\section{LAS COMPAÑÍAS TEATRALES Y LOS TEATROS}

\subsection{Bilingual Foundation of the Arts}

Según la página web de la compañía teatral (http://www.bfatheatre.org/), el compromiso que Bilingual Foundation of the Arts ha tenido con los ciu-

${ }^{1}$ La tesis completa se encuentra en la página web del SELITEN@T, http://www.uned.es/centroinvestigacion-SELITEN@T/index2.html, bajo el enlace, «Estudios sobre teatro». 
dadanos de Los Ángeles, desde 1973, ha sido presentar los mejores ejemplos del legado literario hispano a los públicos hispanohablantes y angloparlantes. La presencia de Bilingual Foundation of the Arts es un componente importante en la producción cultural de Los Ángeles, al ayudar a las diversas culturas de la ciudad a entenderse mejor porque sus producciones enfatizan las semejanzas de la condición humana que nos unen, no las diferencias que nos separan. Dos personas bien conocidas dentro del mundo del teatro fundaron Bilingual Foundation of the Arts: la actriz mexicoamericana Carmen Zapata y la actriz, dramaturga y directora cubana Margarita Galbán. Carmen Zapata ha tenido una carrera muy larga en el mundo del espectáculo. El 2 de octubre del año 2003, la comunidad de Hollywood la honró a ella y a su impacto otorgándole una estrella en Hollywood Walk of Fame, el equivalente al salón de la fama de los que se han destacado en el mundo del entretenimiento (Sauceda, La Opinión, 2003). Margarita Galbán es una premiada directora y adaptadora con renombre mundial. Ha dirigido más de cien producciones, algunas de las cuales han sido bilingües y otras en español e inglés (Fliotsos y Vierow, 2009: 190-196). El rey Juan Carlos les ha otorgado a las dos la Cruz Oficial de la Orden de Isabel la Católica por su compromiso para preservar el legado hispano a través del arte y la literatura. Bilingual Foundation of the Arts ha adquirido una reputación por representar el teatro hispano de la más alta calidad a los ciudadanos de Los Ángeles y California. Sus interpretaciones en español les infunden orgullo a los públicos hispanohablantes y proveen una introducción a la historia rica y elocuente de la cultura hispana a los públicos angloparlantes. Para satisfacer los gustos lingüísticos de estos dos públicos, Bilingual Foundation of the Arts suele poner sus obras en escena en dos formatos. En ocasiones, alterna entre una semana de representaciones en español y una semana en inglés y, en otras, hacen todas las representaciones de una obra en español con sobretítulos proyectados encima del escenario en inglés. En enero de 2007, la compañía estimó que su audiencia anual era de 60.000 personas («Fundación de Los Ángeles promueve la cultura hispana a través del teatro», $w w w$. terra.com.pr/noticias/articulo/html/act724384.htm).

\subsubsection{El Pequeño Teatro de Bilingual Foundation of the Arts / Teatro Carmen Zapata}

Este teatro tiene unos 99 asientos y es el hogar de la compañía teatral, Bilingual Foundation of the Arts. Está ubicado en una sección de Los Ángeles que se conoce como Lincoln Heights. El edificio en el que el teatro se 
encuentra era antes la prisión de Lincoln Heights. El 13 de noviembre del 2005, el nombre del teatro cambió de El Pequeño Teatro de Bilingual Foundation of the Arts a Teatro Carmen Zapata, en honor de una de las fundadoras de la compañía teatral («Carmen Zapata tendrá 'su’ teatro», La Opinión).

\subsubsection{The Los Angeles Theatre Center}

Este teatro (http://thelatc.org/) es propiedad de la Ciudad de Los Ángeles y la compañía teatral, Latino Theater Company, lo dirige. En el año 2006, Latino Theater Company recibió \$4.000.000 de California Cultural and Historic Endowment para que pudieran hacer renovaciones y modernizar el edificio. El teatro abrió de nuevo en 2007. Su propósito es poner en escena obras que reflejan la diversidad de la población de Los Ángeles, realizando representaciones en español, en dos modalidades: la representación entera se da en español o se presenta en español con sobretítulos en inglés.

\subsection{Malayerba}

En el archivo virtual, Artes escénicas, de la Universidad de Castilla-La Mancha (http://artesescenicas.uclm.es/index.php?sec $=$ artis \&id $=118 \&$ PHP SESSID=azhwlexmmzvpjcee), se explica que este grupo teatral se fundó en Quito en el año 1979. Dos años después, la compañía se estableció como una organización sin fines de lucro, llamada Asociación Cultural Taller de Teatro Malayerba. Era un grupo «absolutamente independiente y destinado a la producción de un Teatro Latinoamericano que exprese su realidad en un lenguaje propio». Para lograr dicho lenguaje, se creó el Laboratorio Teatral Permanente, en Quito, donde, en diferentes sitios dentro de la ciudad, se dedicaron a la experimentación teatral y la enseñanza. En el año 1995, después de una gira en Francia, la compañía consiguió suficientes fondos para poder establecerse en un sitio fijo en Quito. En 1996, la Casa de la Cultura Ecuatoriana le otorgó la Medalla Nacional al Mérito Teatral y en el 2001 nació «la revista Hoja de Teatro, bajo la dirección de Daysi Sánchez, como un instrumento para la crítica, la teorización, el pensamiento del teatro». A lo largo de su trayectoria, Malayerba ha demostrado que su identidad como grupo teatral se define a través de la mezcla. Esta mezcla es evidente en su elenco, constituido por «actores profesionales de distintos orígenes, distintas nacionalidades, distintas culturas y procedencias». Ha llevado a escena 21 montajes, tanto dentro de Ecuador como en festivales nacionales e internacionales. También ha tenido una presencia en el cine y la televisión. 
Malayerba vino a Los Ángeles, como parte de Festival Internacional de Teatro de Los Ángeles, FITLA, en el año 2004. Desde el 2002 hasta el 2008, FITLA trajo 30 compañías teatrales de catorce países a Los Ángeles. Estas compañías pusieron 55 obras en escena (Coates, 2012: 903-904). FITLA ayudó a enriquecer la oferta teatral en español en esta ciudad. En los años 2000 y 2001, los únicos años estudiados antes del comienzo de FITLA, los dos grupos principales que representaron obras en español, Bilingual Foundation of the Arts y Grupo de Teatro SINERGIA fueron los que se encargaron de las puestas en escena de 21 de las 23 obras representadas (Coates, 2012: 708).

\subsubsection{Ford Theatre}

Esta entidad (http://www.fordheaters.org/) cree que las puestas en escena que presenta a la comunidad deben representar la riqueza cultural de la ciudad. Busca dar voz a los varios grupos étnicos de Los Ángeles y presentar las culturas de muchas partes del mundo. De esta manera, el público no solo disfruta de un espectáculo teatral de primera calidad, sino que también tiene la oportunidad de entender mejor otra cultura vecina de Los Ángeles. Cuando este teatro tiene puestas en escena en español, utiliza uno de los dos formatos: o son representadas totalmente en español o en español con sobretítulos en inglés. En el año 2002, Ford Theatre recibió \$450.000 de James Irvine Foundation para comenzar Latino Audience Initiative, un intento de traer más producciones con tema latino a Los Ángeles, con el fin de atraer a más latinos al teatro (Shirley, The Los Angeles Times, 2002, http://articles. latimes.com/2002/jun/10/entertainment/et-shirley10). Durante los años 2002-2004, Ford Theatre utilizó este dinero para poner 14 obras en escena en español (Coates, 2012: 82).

\subsection{Teatro de las Américas}

Esta compañía teatral, fundada en el año 1991, está ubicada al noroeste de la ciudad de Los Ángeles, en Oxnard, y la gran mayoría de sus representaciones se ofrecen en esta ciudad. No obstante, de vez en cuando, pone en escena una obra en Los Ángeles. Según su página web (http://teatrodelasamericas.org/), el grupo teatral tiene una visión muy semejante a la de Bilingual Foundation of the Arts. Su propósito es unir a los públicos hispanohablantes y angloparlantes «por medio de producciones teatrales, accesibles a los que hablan inglés y a los que hablan español, dando oportunidades de 
crecimiento, autoconocimiento, reflexión profunda en el pensamiento y en la emoción, para todos los que participan en las producciones y los que asisten a las mismas».

\subsubsection{Frida Kahlo Theater}

Este teatro, de unas 100 sillas, fue fundado en el año 1994. Desde 1994-2001, se conoció como Unity Arts Center. En el 2001, se cambió el nombre al de Frida Kahlo Theater. Es el hogar de una de las compañías teatrales de Los Ángeles que se dedica a la representación de obras en español, Grupo de Teatro SINERGIA (http://fridakahlotheater.org/). Fue bautizado así por la famosa pintora mexicana, Frida Kahlo, sobre la que el Grupo de Teatro SINERGIA ha representado una obra en más de 500 ocasiones a lo largo de 10 años. El formato preferido del Grupo de Teatro SINERGIA es alternar entre una semana de puestas en escena en español y una semana en inglés. Otras compañías teatrales, como Teatro de las Américas, ocasionalmente representan obras en este teatro.

\section{LA CARTELERA}

\subsection{Año 2000}

\subsubsection{La dama duende}

Bilingual Foundation of the Arts interpretó la comedia, La dama duende, escrita por Pedro Calderón de la Barca, los días 13, 14, 15, 16, 27, 28, 29 y 30 de abril y los días 11, 12, 13, 14, 19, 20 y 21 de mayo. Bilingual Foundation of the Arts la representó una semana en español y otra semana en inglés. El horario fue el siguiente: los jueves, viernes y sábados a las 20:00 horas y los domingos a las 15:00 horas. Los precios de las entradas variaron entre 13 dólares estadounidenses la noche antes del estreno oficial, 25 dólares la noche del estreno y 20 dólares en las demás representaciones. Hubo descuentos para personas de la tercera edad, profesores, alumnos y miembros de la industria del entretenimiento. Estas personas pagaron 16 dólares. Agustín Coppola adaptó y dirigió la producción y los intérpretes fueron Sonya Smith, Azalea Correa, Rachel García, Daniel Novoa, Antonia Nesme, José Ramos y Patricia DeLaunay (Coates, 2012: 105-113). 
Veamos algo de su recepción crítica. En una crítica favorable, el crítico alaba tanto al autor de la obra como la puesta en escena. Dice que don Pedro Calderón de la Barca era un «gran filósofo» del Siglo de Oro y que era «capaz de crear personajes (muy) desarrollados», los que, en ocasiones «usa a contrapelo, para sorprender». Un ejemplo que propone para demostrar esta capacidad de sorprender es don Manuel, quien no es el «típico héroe sólido, el gran espadachín que todo lo sabe y que conquista mujeres con seguridad en sí mismo». En cambio, este héroe «no es mejor que quien hace las partes de villano... y en vez de conquistar a mujeres a troche y moche se convierte en juguete de una a quien ni si quiera conoce». Además, el intérprete de don Manuel, Daniel Novoa, representa al personaje «inteligentemente» $\mathrm{y}$ «con un aire de sensibilidad y un aire despistado». Los criados son otro ejemplo de los personajes utilizados para sorprender. Estos, «que tradicionalmente en el teatro de entonces eran más listos que sus amos, acá son uno más tonto que el otro; solo Isabel, criada de doña Ángela — la susodicha 'dama duende'quiere ser pícara y traviesa, pero a menudo se equivoca». Expresa otros elogios como que la puesta en escena tiene un «espíritu lúdico que corre como un quisquilloso río de principio a final de la comedia». En cuanto al elenco, el crítico opina que «muestra estar a la altura de la obra». Menciona específicamente a Eleazar del Valle, Edward Padilla, Patricia DeLauney, Azalea Correa, Antonio Nesme, José Ramos y Sonya Smith. El director, Agustín Coppola, logra «crear una compleja y elaborada trama de movimientos en el desarrollo, demostrando una activa imaginación» y la escenógrafa, Estela Scarlata y el encargado del vestuario, Carlos Brown, también se destacan por su trabajo excepcional (Quintana, La Opinión, 2000).

Otro crítico dice que esta comedia es un ejemplo perfecto de la comedia del Siglo de Oro de España. Recibe una producción adecuada por parte del director Agustín Coppola y, aunque es benignamente graciosa en la primera parte, los actores Edward Padilla y Eleazar del Valle ayudaron a la obra con sus burlas bajas de los sirvientes tontos (Hernández, LA Weekly, 2000).

En otra reseña, la crítica se concentra en el elenco y el director, atacando al guión con energía. La dirección de Agustín Coppola es atrevida y arriesgada, pero cuando una obra tiene un personaje, Rodrigo, interpretado por Eleazar del Valle, que le ruega al público que no tire cosas al escenario para demostrar su disgusto, no está exactamente fuera de lugar (Monji, Los Angeles Times, 2000, http://articles.latimes.com/2000/apr/28/entertainment/ ca-24213). 
Los elogios continúan en otra crítica. En su opinión, la gente, que no presencia La dama duende en Bilingual Foundation of the Arts, está perdiendo una buena oportunidad. El director de la obra Agustín Coppola ha añadido mucho misterio travieso e intriga a esta obra clásica de una de las figuras principales del Siglo de Oro, Pedro Calderón de la Barca. Para ella, la bella y talentosa Sonya Smith, una estrella de telenovelas en su país natal de Venezuela, ofrece una interpretación estupenda como la protagonista, doña Ángela. Otras interpretaciones igualmente emocionantes son las de Antonio Nesme y José Ramos, quienes, como los hermanos vigilantes de Ángela, han determinado protegerla de los avances de los galanes que, en la opinión de ellos, no la merecen. También, brillante en su representación de Rodrigo, el narrador de la obra, Eleazar del Valle, lleva su papel a la máxima altura y es el que exige la atención del público. Estela Scarlata ofrece un escenario extraordinario y el maestro del vestuario, Carlos Brown, recibe ayuda con la coordinación del vestuario por Azalea Correa y Beatrissa Radel. En su opinión, no se debe perder esta graciosísima producción agradable (Soto, Eastern Group Publications, 2000).

\subsubsection{Don Quijote, la última aventura}

Los días 21, 22, 23 y 24 de septiembre y los días 5, 6, 7, 8, 19, 20, 21, 22, 26, 27, 28 y 29 de octubre, Bilingual Foundation of the Arts representó la comedia, Don Quijote, la última aventura, una adaptación de la obra, Don Quijote de la Mancha, de Miguel de Cervantes Saavedra, en Los Angeles Theatre Center. Margarita Galbán y Lina Montalvo adaptaron la obra y Galbán también la dirigió. Bilingual Foundation of the Arts varió entre una semana de representaciones en español y una semana en inglés. El horario típico fue los jueves, viernes y sábados a las 20:00 horas y los domingos a las 15:00 horas. Los precios de las entradas variaron entre 30 dólares estadounidenses la noche del estreno, 22 dólares los jueves y 24 dólares en las demás representaciones. Hubo descuentos para personas de la tercera edad, profesores, alumnos y miembros de la industria del entretenimiento. Estas personas pagaron 17 dólares los jueves y 19 dólares durante el fin de semana. El elenco incluyó a Jaime Arze, Rey López, Agustín Coppola y Paola Bontempi. Estela Scarlata se encargó de la escenografía y Carlos Brown diseñó el vestuario (Coates, 2012: 113-125).

Sobre su recepción crítica exponemos lo siguiente. En una reseña negativa, el crítico compara esta producción con Bodas de sangre, representada por Bilingual Foundation of the Arts en el año 1999. La calidad que presen- 
ció en la obra de García Lorca no se repitió en Don Quijote, la última aventura. En opinión del crítico, el trabajo es tan arduo que se puede sentir el esfuerzo, cada onza del esfuerzo, hasta los últimos diez minutos. Para el clímax, la adaptación de la directora Margarita Galbán ofrece un duelo diseñado cuidadosamente entre León (Jaime Arze), un actor enamorado del papel del caballero andante de Cervantes, y el realista severo don Luis (Rey López), el enemigo jurado de León. Los que atestiguan el duelo se envuelven en él. Cuando León levanta la cabeza para recordarnos el hecho de que solo es una representación — que el Quijote seguirá viviendo- la presunción de la producción llega a su máxima eficacia. No obstante, según el crítico, la llegada a este punto cuesta mucho trabajo. La escenografía, un mercado español del siglo XVI diseñado por Estela Scarlata, es agradable y el vestuario diseñado por Carlos Brown es notable. Sin embargo, las adaptadoras Galbán y Lina Montalvo viran, inciertamente, entre escenas entrelazadas. La calidad de las bromas está forzada. Por un lado, es gracioso ver al galán Arze usar paja como un bigote y barba temporal, pero, por otro lado, cansa la segunda vez que lo hace.

El crítico opina que la raíz del problema radica en el tratamiento del texto original de Cervantes, el cual tiene un montón de bromas bajas. El autor escribió una parodia imprudente de la caballerosidad literaria en dos tomos. Sin embargo, en este país, la reputación de esta gran novela satírica se ha disminuido a causa de, entre otras cosas, el musical Man of La Mancha. De modo semejante, en sus apuntes en el programa de mano, la presidenta de Bilingual Foundation of the Arts, Carmen Zapata, enfatiza la importancia de los valores humanos y los altos estándares morales de la novela, en la que Cervantes logró parodiar, invertir, comentar y encarnar el idealismo romántico. Es una hazaña formidable. Pero si se va directamente al idealismo, se pierde lo demás. A menudo, esta producción pierde y este tipo de comedia no es suficientemente creativo a lo largo de la producción, hasta llegar a esos últimos minutos emocionantes, cuando se ciñen las espadas. Es solamente a través de estos últimos gestos dramáticos que Don Quijote nos lleva completamente a sus realidades duales: las aventuras de Quijote por encima de las de León (Phillips, The Los Angeles Times, 2000, http://articles.latimes.com/2000/oct/03/entertainment/ca-30634).

En una reseña positiva, el crítico expresa que el guion está bien realizado y el lenguaje es claro y gratificante. A la vez, mantiene una cierta alteza. El elenco comparte la exuberancia de crear teatro. Constata que Arze interpreta a León con una teatralidad compleja que no es suficiente para explicar el trabajo estupendo que lleva a cabo. Galbán dirige con 
una energía contagiosa, por eso no hay actores menores en la última fila. Entretiene observar lo que ocurre en cada momento. Galbán también incluye unos detalles tiernos: se toca una mandolina en armonía perfecta con la música; se tira una cebolla a través del escenario y dentro de un vaso. El agradable escenario lleno de colores de Estela Scarlata incluye un puente sobre el que la gente del pueblo camina. El vestuario de Carlos Brown reluce bajo la iluminación de colores de joyas de Robert Fromer. La coreografía de Mari Sandoval incorpora el flamenco y el director de peleas Ray Michaels Quiroga ha diseñado duelos y caídas realistas (Margolies, Backstage, 2000).

Otra crítica también elogia la puesta en escena. En su opinión, Margarita Galbán dirige la obra con una mano segura. Un problema que nota es que este Don $Q$ lleva al público en un acortado viaje teatral, encantador a los ojos pero no a los oídos. ¿Por qué resulta que todos los intérpretes de BFA suelen hablar en voz demasiado alta, es decir, gritar? Los matices caen al abismo, a pesar del hecho de que el teatro no es muy grande, lo cual ayuda a que la cacofonía domine a veces. Dejando este problema de lado, la crítica vuelve a las alabanzas. Jaime Arze interpreta al actor León con pasión y Agustín Coppola encarna a su amigo, Alonso, también con celo. A lo largo de la representación, se convierten en Don Q y Sancho P, con la bella Paola Bontempi representando a la mujer noble Aldonza, quien, claro, es la Dulcinea de nuestro héroe. El escenario de Estela Scarlata, una típica plaza española, con cajas de flores colgadas de las ventanas y paja tirada por todo el escenario, es eficaz, haciendo que se desee vivir en tiempos más sencillos. Todo divierte, ayudado por el vestuario auténtico de Carlos Brown con pantalones de terciopelo, muselinas de campesinos y faldas de muchos colores; la iluminación de Robert Fromer es muy apropiada, la coreografía de gitanos de Mari Sandoval tiene espíritu y las peleas de espadas de Ray Michaels Quiroga son creativas. Además, son dignos de mención: Héctor de Alba y Alejandra Flores al interpretar a los padres de León; Berta Holguín es la graciosísima Jimena y Rey López es el malvado don Luis (Looseleaf, Los Angeles Downtown News, 2000).

A otro crítico le agrada que esta «inteligente y sofisticada» puesta en escena utilice elementos del metateatro: «Nos muestra a un actor que tiene una «versión original» de Don Quijote, escrita para el teatro por el mismo Cervantes. El resultado es una bella comedia de una obra, dentro de una obra, de un hombre obsesionado con las obsesiones de otro hombre». El elenco es buenísimo y menciona específicamente a los intérpretes principales, Jaime Arze (Quijote), Agustín Coppola (Sancho) y Paola Bontempi 
(Dulcinea): «Los tres — nos dice— son conmovedores, creíbles y presentan personajes muy interesantes que a uno le gustaría conocer en la vida real». Además, son notables Rey López y Antonio Nesme. Rey es tan bueno en su papel del enemigo «que hay que resistir la tentación de subirse al escenario y sacarlo a golpes» y el «sentido cómico» de Nesme es excelente. El crítico espera que «la Fundación Bilingüe de las Artes tenga en el futuro más aventuras como esta» (Fernández, Mundo LA, 2000).

\subsection{Año 2001}

\subsubsection{Calderón enamorado}

Bilingual Foundation of the Arts representó la adaptación de varios textos de Pedro Calderón de la Barca, Calderón enamorado, el 16 de julio del 2001 a las 20:00 horas en su Pequeño Teatro. José Ruano de la Haza realizó la adaptación y Margarita Galbán dirigió esta producción, la cual formó parte del festival, Teatro Leído. El precio de la entrada fue de 3 dólares estadounidenses (Coates, 2012: 152). Aunque Calderón enamorado no se volvió a poner en escena en otra temporada durante los años estudiados, el propósito del festival, Teatro Leído / New Works Festival, era dar a Bilingual Foundation of the Arts la oportunidad de poner a prueba en una o dos representaciones obras que se pensaban poner en cartelera durante seis $\mathrm{u}$ ocho semanas en un año futuro. Por ejemplo, la obra Juventud, divino tesoro, del autor Raúl de Cárdenas, formó parte del festival Teatro Leído en este año también, y fue representada el 9 de julio (Coates, 2012: 152). Después, se representó en el 2002, desde el 11 de abril hasta el 26 de mayo (Coates, 2012: 198-209).

\subsection{Año 2002}

\subsubsection{El Alcalde de Zalamea}

En su Pequeño Teatro, Bilingual Foundation of the Arts puso en escena la comedia de Pedro Calderón de la Barca, El Alcalde de Zalamea, los días 19, 20, 21 (con dos representaciones este día) y 22 de septiembre, los días 3, 4, 5 (con dos representaciones este día), 6 17, 18, 19 (con dos representaciones este día) y 31 de octubre y los días 1,2 (con dos representaciones este día) y 3 de noviembre. Bilingual Foundation of the Arts varió las puestas en escena: una semana en español y otra en inglés. El horario típico fue los 
jueves y los viernes a las 20:00 horas, los sábados a las 16:00 y 20:00 horas y los domingos a las 15:00 horas. Los precios de las entradas variaron entre 17 dólares estadounidenses la noche antes del estreno oficial, 32 dólares la noche del estreno, 24 dólares estadounidenses los jueves y 26 dólares durante el fin de semana. Hubo descuentos para personas de la tercera edad, profesores, alumnos y miembros de la industria del entretenimiento. Estas personas pagaron 19 dólares los jueves y 21 dólares durante el fin de semana. Margarita Galbán y Lina Montalvo realizaron la adaptación y Galbán también dirigió la producción. El elenco incluyó a Adrian Benedetti, Elizabeth Cuevas, Héctor de Alva, Eleazar del Valle, Gabriel Flores, Gregory G. Giles, Yvonne Gutiérrez, Scarleth Hauffen García, Aaron Hernández, Hugi Isaac, Daniel Light, Kiko Mahecha, Juan Mendoza, Susan Pineda, Ray Michaels Quiroga, Ernesto Miyares, Kirstie Neilson, Edward Padilla, Verónica Stocker, Glenda Torres, Manuel Valdivia e Ignacio Zamudio. Las escenas musicales incluyeron algunos textos del mismo Pedro Calderón de la Barca y piezas originales de Kiko Mahecha. Estela Scarlata diseñó la escenografía, Robert Fromer se ocupó de la iluminación y Carlos Brown arregló el vestuario (Coates, 2012: 220-241).

Con respecto a su recepción crítica, señalaremos lo siguiente. A uno de los críticos le gustó que la representación comience en el lobby del teatro: «Esta es una gran idea — señala — porque aumenta el «espacio mágico» del escenario y lo proyecta fuera de la sala hasta la misma puerta del local. Esta es una forma más de envolver al público e invitarlo a ser parte de la obra». El crítico dice que la puesta en escena es «un placer... con la visión artística y el buen gusto que la directora Margarita Galbán le da a todo lo que hace; inclusive la escena de la violación y la imagen literal de «la virgen caída» es impecable». Los representantes principales, Ernesto Miyares (Pedro Crespo, el Alcalde de Zalamea) y Ray Michaels Quiroga (don Lope), se destacan por su «excelente voz, presencia y lenguaje corporal». También, las actuaciones de Daniel Light (el Capitán), Adrián Benedetti (Juan Crespo) y Kiko Mahecha (Rebolledo) son «sólidas». Para el crítico, Verónica Stocker (Isabel) y Glenda Torres (La Chispa) también son excelentes. Stoker es «comedida y refrenada, como amerita una mujer virtuosa del siglo $17 »$, mientras Torres es «bulliciosa y pendenciera» (Fernández, Mundo LA, 2002).

A otra crítica no le agrada la manera en la que la puesta en escena comienza. Hay travesuras y mucha música folclórica y, en su opinión, es demasiado amplia. No obstante, después se profundiza cuando la trama se hace más seria. Se interpreta la violación de Isabel y sus consecuencias con una veracidad brutal, con algunos fallos, pero con mucha pasión. Con- 
cluye esta reseña indiferente señalando que para el público moderno tal vez sea difícil aceptar la caída de Isabel debido a su «crimen», pero su situación todavía despierta nuestra simpatía como también lo hace el valiente intento de vengar el agravio hecho por su padre. Sería bueno bajar la intensidad de algunas interpretaciones demasiado dramáticas, aunque la obra presenta una rara oportunidad de ver una representación que todavía es capaz de cautivarnos, como un drama y como un barómetro de cambios en las normas sociales (Foley, The Los Angeles Times, 2002).

En otra reseña, positiva, el crítico explica que el texto trata sobre el orgullo, la arrogancia, la lujuria y el poder y no tiene nada que ver con el gobierno estadounidense. Este drama de Pedro Calderón de la Barca, escrito en el siglo XVII, es un ejemplo excelente del concepto de la violación de la virtud femenina y lo que su honra sufre mientras que la burocracia decide cómo castigar al agresor. La adaptación, de Margarita Galbán y Lina Montalvo, tiene una excelencia en cada escena. El vestuario, la música y el canto ayudan a establecer la necesidad del pueblo de sentir el honor y el orgullo (Ruiz, Entertainment Today, 2002).

Finalmente, otra crítica tiene palabras positivas tanto para la obra como para la puesta en escena. Describe la pieza como una poderosa comedia, escrita por el dramaturgo español Pedro Calderón de la Barca, que tiene que ver con las vidas de los familiares de un alcalde en un pueblo pequeño de España y las consecuencias devastadoras del abuso del poder y la brutalidad de los aguerridos soldados españoles. Es un vistazo a otra época y lugar donde el falso sentido del honor de un soldado eclipsa la violación de la inocencia. En cuanto a la puesta en escena, dice que mientras la directora Margarita Galbán incluye el humor dentro del caos y la maldad que penetran la obra, los personajes nunca pierden el enfoque del público. En un momento, hay burlas y, posteriormente, se presentan razones para enfadarse con los antagonistas y sentir lástima por los que se hieren. Ernesto Miyares inteligentemente representa al alcalde que se enfrenta a la peor de las pesadillas, el sinvergüenza Capitán don Álvaro de Ataide, brillantemente interpretado por Daniel Light (Soto, Eastern Group Publications, 2002).

\subsection{Año 2003}

\subsubsection{Los clásicos... enredos}

Los días 3, 4, 5 (con dos representaciones este día), 6, 17, 18, 19 (con dos representaciones este día) y 20 de abril y los días $1,2,3$ (con 
dos representaciones este día), 4, 15, 16, 17 (con dos representaciones este día) y 18 de mayo, Bilingual Foundation of the Arts representó la adaptación de varios textos de Pedro Calderón de la Barca, Sor Juana Inés de la Cruz, Lope de Vega y Juan Ruiz de Alarcón, Los clásicos... enredos, en su Pequeño Teatro. Margarita Galbán y Lina Montalvo llevaron a cabo la adaptación y Galbán también dirigió la producción. Bilingual Foundation of the Arts varió entre una semana de producciones en español y una semana en inglés. El horario típico fue los jueves y los viernes a las 20:00 horas, los sábados a las 16:00 y 20:00 horas y los domingos a las 15:00 horas. Los precios de las entradas variaron entre 24 dólares estadounidenses los jueves y 26 dólares durante el fin de semana. Los intérpretes fueron Carlos Oliva, Alejandra Flores, Sonya Smith, Jaime Arze, Azalea Correa, Rachel García, Ernesto Miyares, Antonio Nesme, Ray Michaels Quiroga, Verónica Stocker, Glenda Torres, Julia Estacolchic, Armando Heredia, Gonzalo Suárez, Natalia Waldhoru e Ignacio Zamudio (Coates, 2012: 267-278).

Por lo que concierne a su recepción crítica apuntaremos lo siguiente. En opinión favorable de uno de los críticos, estas textualidades «son una clara muestra de lo divertidos que pueden ser los clásicos y, además, de la importancia de los temas que tratan y justifican su permanencia en la cultura universal». Demuestran la importancia de las obras del Siglo de Oro al explorar «cuestiones básicas relacionadas con la incertidumbre de la mente humana en la percepción de la realidad: el ser y el parecer». También le agrada la manera en la que las adaptadoras Margarita Galbán y Lina Montalvo han combinado las selecciones de las obras. El resultado es «una producción de alto nivel profesional y, sobre todo, llena de vida». Sobre el elenco: el tener que representar a varios personajes de obras distintas «no inhibe a estos actores, sino que parece impulsarlos a acentuar la definición de los caracteres de turno y sus cuitas». Nombra a los actores que dan «brillo» a la representación: Jaime Arze, Azalea Correa, Alejandra Flores, Rachel García, Ernesto Miyares, Antonio Nesme, César Oliva, Ray Michaels Quiroga, Sonya Smith, Verónica Stocker, Julia Estacolchic, Armando Heredia, Gonzalo Suárez, Natalia Waldhoru e Ignacio Zamudio. Y termina: «La práctica escenografía» de Estela Scarlata «se adapta mágicamente a las distintas historias, y el vestuario de Carlos Brown logra alcanzar el mismo grado de ubicuidad» (Quintana, La Opinión, 2003). 


\subsection{Año 2004}

\subsubsection{De cómo moría y resucitaba Lázaro, el Lazarillo}

Los días 28, 29, 30 y 31 de octubre y el 11 de noviembre, Malayerba interpretó la obra de Arístides Vargas, De cómo moría y resucitaba Lázaro, el Lazarillo, una dramatización de la novela española, La vida de Lazarillo de Tormes y de sus fortunas y adversidades, en Ford Theatre. El adaptador, Arístides Vargas, dirigió la producción y formó parte del elenco, junto con Charo Francés y Gerson Guerra. José Lino Suntaxi se ocupó de la iluminación y José Rosales realizó el vestuario. La puesta en escena del 31 de octubre empezó a las 15:00 y las representaciones que restan empezaron a las 20:00 horas. El precio de la entrada fue de 15 dólares estadounidenses y 12 dólares para alumnos y niños menores de doce años. Esta producción formó parte del Festival Internacional de Teatro de Los Ángeles. Esta obra se representó exclusivamente en español (Coates, 2012: 366-371).

Sobre su recepción crítica indicaremos que uno de los críticos manifestaba que esta puesta en escena es una «hazaña». El elenco es fenomenal y «nos provee de un espectáculo sensacional». Específicamente, Charo Francés «tiene el don singular de conquistar al público en forma inmediata con una generosa capacidad de empatía, cualquiera sea el sentimiento que despliegue en su escena» y Gerson Guerra «es un actor de plenos recursos físicos y expresivos, totalmente concentrado en cada uno de los papeles que le tocan». También, Arístides Vargas es excepcional en su trabajo como adaptador y director. Al fin y al cabo, «este espectáculo en desarrollo es teatro vivo, en toda su seductiva pureza, quizá más que una obra terminada con todos sus puntos y comas, publicada y aspirando a premio» (Quintana, La Opinión, 2004).

\subsection{Año 2005}

\subsubsection{Don Quijote, la última aventura}

Esta comedia, adaptada de la novela de Miguel de Cervantes Saavedra, se puso en escena en el Pequeño Teatro de Bilingual Foundation of the Arts durante el otoño de 2005. Se sabe que Bilingual Foundation of the Arts varió entre una semana de representaciones en español y una semana en inglés, sin embargo, debido a una falta de fuentes, se desconocen las fechas precisas de las puestas en escena en español. Por eso, se aproximan las fe- 
chas de representación en español: el 30 de septiembre, los días 1, 2, 13, 14, $15,16,27,28,29$, y 30 de octubre y los días $10,11,12$ y 13 de noviembre. Antes de la última representación, el nombre del teatro cambió de El Pequeño Teatro de Bilingual Foundation of the Arts a Teatro Carmen Zapata. El horario fue los jueves, viernes y sábados a las 20:00 horas y los domingos a las 15:00 horas. Margarita Galbán y Lina Montalvo realizaron la adaptación y Galbán también dirigió la producción. Estela Scarlata diseñó la escenografía, Carlos Brown arregló el vestuario, Mari Sandoval fue la coreógrafa y Edward Motts se encargó de la iluminación. Los intérpretes fueron Jaime Arze, Eleazar del Valle, Ricardo Rocha, Nallely Cardona, Michelle Castillo, Raúl Ávila, Ariana Estrada, David Carreño, Kiko Mahecha, J. Pérez Amor, Gerardo Arenas, Gerardo Toro, Lina Montalvo, Yesika Baker, Agnacio Zamudio, Isabel Arze, Davina Ferreira, Flor de María Chahua, Abraham Morales y Henry Madrid (Coates, 2012: 408-423).

Según la crítica, la representación no es sobre Don Quijote ni su última aventura. No obstante, la producción es bastante gozosa, a pesar de que Don Quijote y Sancho nunca pisen el escenario. Debido al hecho de que no se mantiene fiel al texto original, es mejor para un público que ya conozca bien la novela. La única manera en la que el espectador puede comparar, contrastar y decidir en los aspectos positivos y negativos de la interpretación es si se tiene en cuenta la versión original. De otra manera, la puesta en escena se convierte en un comentario sobre varios asuntos sociales, lo cual es apropiado para la comunidad en general en una ciudad como Los Ángeles, pero no es ventajoso para el público académico que trabaja con el legado literario para determinar el valor artístico de una representación (Gasior, Comedia Performance, 2008).

\subsection{Año 2006}

\subsubsection{El burlador de Sevilla y convidado de piedra}

En Frida Kahlo Theater, Teatro de las Américas interpretó esta tragedia de Tirso de Molina, el 1 de abril a las 14:00 horas. La obra se representó en español con sobretítulos proyectados arriba del escenario en inglés. Lourdes Solórzano dirigió la producción y los actores fueron Jesús Ochoa, Ricardo Ayala, Hugo Carreón, Aldo Cecena, Jim Valdez, Rigoberto Guízar, Benjamín Girón, Rogelio Guerrero, Jesús Ruesga, Pat Casiano, Rocío Martínez, María Magdalena Esparza, Mayela Cortéz, José Aguilar, David Tapia, Lizette Rodríguez, Juan Esparza y Aarón René Guízar. Susan Hernández diseñó 
la escenografía y Edward Dávila se encargó de la iluminación. El precio de la entrada fue de 10 dólares estadounidenses y 7 dólares para personas de la tercera edad y estudiantes (Coates, 2012: 447).

\subsection{Año 2007}

\subsubsection{El anzuelo de Fenisa}

Bilingual Foundation of the Arts puso en escena El anzuelo de Fenisa, una comedia escrita por Lope de Vega, en el Teatro Carmen Zapata, los días 15, 16, 17 (con dos representaciones este día), 18, 22, 23, 24 (con dos representaciones este día) y 25 de febrero y los días $8,9,10$ (con dos representaciones este día), 11, 15, 16, 17 (con dos representaciones este día), 18, 22, 23, 24 (con dos representaciones este día) y 25 de marzo. El horario fue los jueves y viernes a las 20:00 horas, los sábados a las 16:00 y 20:00 horas y los domingos a las 15:00 horas. Bilingual Foundation of the Arts alternó una semana en español y otra en inglés. Los precios de las entradas variaron entre 35 dólares estadounidenses la noche del estreno, 27 dólares los jueves y 28 dólares durante el fin de semana. El elenco incluyó a Verónica Stocker, Rachel García, Annabel Turrado, Ricardo Rocha, Alejandro Jiménez, Erik S. Revolorio, Raúl Ávila, José Martínez, Raúl Rene Peraza, Daniel Restrepo, Ignacio Zamudio, RF Rodríguez, Francisco García, Henry Madrid, Jessica Silvetti, Lucía Zepeda y David García. César Oliva Bernal llevó a cabo la adaptación y dirigió la producción para Bilingual Foundation of the Arts. Estela Scarlata diseñó la escenografía, Edward Motts se encargó de la iluminación y Carlos Brown del vestuario (Coates, 2012: 494-505).

\subsection{Año 2009}

\subsubsection{La vida es sueño}

Se puso en escena la obra de Pedro Calderón de la Barca, La vida es sueño, el 15 de junio a las 20:00 horas en Teatro Carmen Zapata. César Oliva Bernal dirigió la producción para Bilingual Foundation of the Arts. El precio de la entrada fue de 5 dólares estadounidenses. Esta interpretación formó parte de New Works Festival (Coates, 2012: 631). 


\section{CONCLUSIONES}

En primer lugar, destacaremos que no se puso en escena en Los Ángeles ninguna obra del teatro áureo español en los años 2000 y 2010. Hubo representaciones de obras del Siglo de Oro en nueve de los once años estudiados. También parece que la crítica apreció estas puestas en escena, se escribieron críticas sobre cinco, el 62,5\% de las ocho obras representadas. Este porcentaje contrasta bien con el de todas las obras que fueron criticadas, 76 de las 182 piezas, el 41,76\% (Coates, 2012: 1054). Además, de las quince críticas escritas sobre estas representaciones, solamente una es decididamente negativa, dos presentan una valoración indiferente y las doce que restan son positivas. De las tres obras no criticadas, dos, Calderón enamorado y El burlador de Sevilla y convidado de piedra, solamente se pusieron en escena en una ocasión. La única obra representada en más de una ocasión que no recibió crítica alguna fue El anzuelo de Fenisa de Lope de Vega.

No obstante, no se puede decir que la presencia del teatro del Siglo de Oro español en Los Ángeles sea fuerte. De las 182 obras representadas, solamente ocho pertenecen al teatro áureo, el 4,40\%. Estas ocho obras se pusieron en escena en 118 ocasiones, el 6,61\% de todas las representaciones. También, cuatro de estas ocho obras, Calderón enamorado, Los clásicos... enredos, Don Quijote, la última aventura y De cómo moría y resucitaba Lázaro, el Lazarillo, son adaptaciones basadas en obras del Siglo de Oro, pero no son los textos originales completos.

¿Qué se puede deducir de todo esto? Hay dos conclusiones posibles, según estudié en mi tesis de doctorado. Primero, las tres obras más representadas durante los once años estudiados son Too Many Tamales, puesta en escena en 181 ocasiones, una obra sobre las celebraciones navideñas de una familia hispana que reside en los Estados Unidos; Las mujeres de Juárez, puesta en escena en 169 ocasiones, una obra que trata de homicidios que han ocurrido en la ciudad fronteriza de Juárez, México y Frida Kahlo, puesta en escena en 108 ocasiones, una biografía sobre la famosa artista mexicana (Coates, 2012: 712). Se ve que hay una preferencia por obras más modernas que hablan de eventos y personas que los hispanos de Los Ángeles reconocen.

Segundo, de los hispanos que residen en Los Ángeles, el 57\% nació en los Estados Unidos y el $43 \%$ llegó al país como inmigrantes (Hayes-Bautista, 2004: 94). Los que nacieron en los Estados Unidos se identificarían más con la literatura de este país o la del país de descendencia de su familia. De los hispanos de los Estados Unidos, solamente el 1,3\% tiene una conexión 
directa con España («The Hispanic Population: 2010, http://www.census. gov/prod/cen2010/briefs/c2010br-04.pdf). En cuanto a los inmigrantes, Josefina López, directora artística del teatro Casa 0101 en Los Ángeles, señala que es difícil atraer a estos al teatro porque acuden "'más a la iglesia, ese es su teatro.... Al final terminamos compitiendo por las almas y el estacionamiento', agrega refiriéndose a la Iglesia Victoria Alianza que está frente a su local» (Carrión, Hoy Los Angeles, 2012, http://www.hoylosangeles.com/ news/2012/mar/30/teatro-latino-en-l-nada-contracorriente/).

A la vez, obras de autores de 25 países se representaron en estos años y España fue el segundo país representado. Debido al hecho de que las compañías teatrales y teatros utilizan un formato que alterna entre una semana de interpretaciones en español y una en inglés o sobretítulos proyectados arriba del escenario en inglés, todos los residentes de Los Ángeles pueden disfrutar de las representaciones. Esta tendencia es la que facilita tanta diversidad en el teatro representado en español en Los Ângeles. Un aspecto importante de esta diversidad es la presencia del teatro del Siglo de Oro.

¿Cómo se compara la presencia del teatro áureo español en Los Ángeles con la que existe en otras ciudades? Como ya se mencionó en la introducción, este artículo es una continuación de la sección monográfica que aparece en la edición número quince de Signa, Puestas en escena de nuestro teatro áureo en algunas ciudades españolas durante los siglos XIX y XX (Aragón, 2006: 11-186). El Dr. José Romera Castillo detalla una gran parte de esta en Pautas para la investigación del teatro español y sus puestas en escena, específicamente en el capítulo cuatro, «Puestas en escena de obras áureas en diversos lugares de España en la segunda mitad del siglo XIX» (2011: 141-171) y el capítulo cinco, «Obras de Lope de Vega en algunas carteleras de provincias españolas (1900-1936)» (2011: 173-184). Tanto en la sección monográfica de Signa como en el libro, se ve que «el teatro clásico ocupa un lugar reducidísimo en las carteleras de las ciudades estudiadas». El profesor Romera Castillo explica que esta situación en España se debe a que «el teatro de nuestros autores clásicos, además de no ser elegido por las compañías por requerir presupuestos más elevados y condicionamientos técnicos importantes — de los que no carecían en general los espacios escénicos provinciales de la época-, no contaba con las preferencias del público» (2011: 184).

En Los Ángeles vemos una situación semejante, al encontrar pocas representaciones de obras del Siglo de Oro debido a una preferencia por obras más modernas que hablan de eventos y personas que los hispanos de la ciu- 
dad reconocen. No obstante, ni en Los Ángeles ni en las ciudades españolas estudiadas, «no cayeron en un olvido total autores y obras de nuestro teatro áureo, que bien ha valido la pena tener en cuenta aquí, tanto por su valor artístico como por su función cultural y social» (Romera, 2011: 171).

\section{REFERENCIAS BIBLIOGRÁFICAS}

ARAGÓN GONZÁLEZ, Irene (ed.) (2006). «Puestas en escena de nuestro teatro áureo en algunas ciudades españolas durante los siglos XIX y XX». Signa. Revista de la Asociación Española de Semiótica 15, 11-186 $<$ ht tp://cervantesvirtual.com/servlet/SirveObras 105818507811614984197857/index.htm>.

«Carmen Zapata tendrá 'su' teatro». La Opinión, 8 noviembre 2005. LexisNexis. Consultado en Internet, el 17 de mayo de 2012.

CARRIÓN, Andrea (2012). «Teatro latino en LA nada contracorriente». Hoy Los Angeles, 30 marzo <http://www.hoylosangeles.com/news/2012/ mar/30/teatro-latino-en-l-nada-contracorrienters. Consultado en Internet, el 9 de agosto de 2012.

COATES, John Benjamin (2012). El teatro representado en español en Los Ángeles durante los años 2000-2010. Tesis doctoral dirigida por José Romera. Madrid: Universidad Nacional de Educación a Distancia $<$ http://www.uned.es/centro-investigacion-SELITEN@T/pdf/John_B_ Coates.pdf.>.

FERNÁNDEZ, Gustavo (2000). «Don Quijote una aventura teatral de la Fundación Bilingüe de las Artes». Mundo LA, 19 octubre, 24.

- (2002). «El Alcalde de Zalamea: El eterno duelo entre la ley civil y la ley militar». Mundo LA, 10 octubre, 28.

FLIOTSOS, Anne y VIEROW, Wendy (2009). American Women Stage Directors of the Twentieth Century. Illinois: University of Illinois Press.

FOLEY, F. Kathleen (2002). «Changing Mores on View in a Spanish Classic». The Los Angeles Times, 4 octubre, F21.

GASIOR, Bonnie (2008). «Theater Review of Don Quijote, la última aventura». Comedia Performance, 191-198.

HAYES-BAUTISTA, David E. (2004). La nueva California: Latinos in the Golden State. Berkeley y Los Ángeles: University of California Press.

HERNÁNDEZ, Martín (2000). «The Phantom Lady». LA Weekly, 28 abril, s.p. 
LOOSELEAF, Victoria (2000). «The Last Adventure». Los Angeles Downtown News, 9 octubre, s.p.

MARGOLIES, Dany (2000). «Don Quijote: The Last Adventure». Backstage, 5 octubre, s.p.

MONJI, Jana J. (2000). «'Phantom Lady’ Romps Into 17th Century Spain With Gusto». The Los Angeles Times, 28 abril <http://articles.latimes. com/2000/apr/28/entertainment/ca-24213>. Consultado en Internet, el 9 de enero de 2012.

PHILLIPS, Michael (2000). «This New 'Don Quixote' Is Still Chasing After Illusions». The Los Angeles Times, 3 octubre. <http://articles.latimes. com/2000/oct/03/entertainment/ca-30634>. Consultado en Internet, el 9 de enero de 2012.

QUINTANA, Hugo (2000). «Noches de ronda». La Opinión, 24 abril, D1.

- (2003). «No hay clásico como el que divierte». La Opinión, 14 abril. LexisNexis. Consultado en Internet, el 17 de mayo de 2012.

- (2004). «Placer de juglares». La Opinión, 30 octubre. LexisNexis. Consultado en Internet, el 22 de mayo de 2012.

ROMERA CASTILLO, José (2011). Pautas para la investigación del teatro español y sus puestas en escena. Madrid: UNED.

RUIZ, José (2002). «The Mayor of Zalamea Bilingual Foundation of the Arts». Entertainment Today, 4 octubre, s.p.

SAUCEDA, Isis (2003). « Viva Carmen Zapata!». La Opinión, 3 octubre. LexisNexis. Consultado en Internet, el 17 de mayo de 2012.

SHIRLEY, Don (2002). «Importing Latino Theater». Los Angeles Times, 10 junio <http://articles.latimes.com/2002/jun/10/entertainment/et-shirley10>. Consultado en Internet, el 9 de marzo de 2012.

SOTO, Rose (2000). «The Phantom Lady». Eastern Group Publications 4 mayo, A4.

- (2002). «East LA \& Beyond: El Alcalde de Zalamea». Eastern Group Publications, 17 octubre, 4.

Recibido el 8 de junio de 2013.

Aceptado el 19 de septiembre de 2013. 
